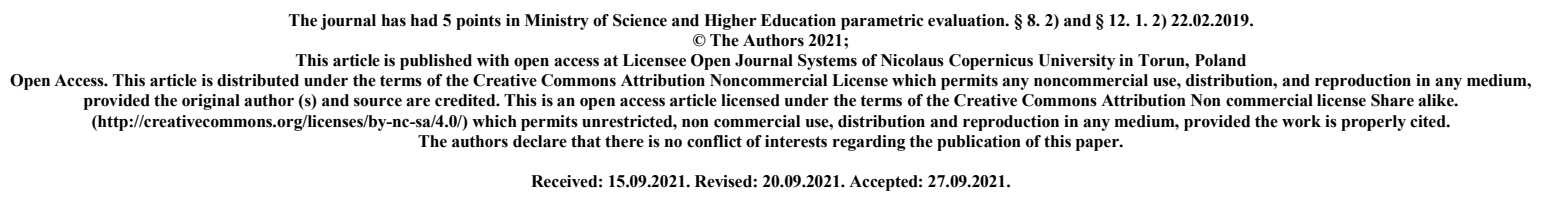

\title{
Epistaxis etiology and treatment
}

\section{Marcin Kożuchowski' ${ }^{1}$, Monika Prylińska ${ }^{1}$}

1.Department of Physiology of Physical Effort and Functional Anatomy, Collegium Medicum in Bydgoszcz, Nicolaus Copernicus University in Toruń, Poland

\begin{abstract}
Epistaxis occurs in approximately $60 \%$ of the general population. Every tenth person requires treatment in a hospital setting. Epistaxis occurs in all age groups, reaching its peak in children around 10 years of age and in adults around 80 years of age. The frequency and intensity of epistaxis are anatomically based on numerous vascular connections. The cause of epistaxis in most cases can be determined on the basis of a detailed history and physical examination. There are a number of treatments available for nose bleeds. From widely available methods such as nasal tamponade to more advanced endovascular procedures.

Key words: Epistaxis, Epistaxis treatment, Epistaxis etiology, Epistaxis management
\end{abstract}




\section{Introduction}

Epistaxis is one of the more common emergencies among otolaryngological patients. It is estimated that this condition affects approximately $60 \%$ of the general population, but every tenth of them seek medical help. In terms of age, epistaxis reaches its peak in two age groups: children under the age of 10 and adults between the ages of 70 and 80 . In terms of gender, men are more likely to experience nosebleeds than women [9], [3], [4]. This disease is very often selflimiting. Therefore, some patients can cope with bleeding on their own. And they come for help when affliction frequently recurs or intensifies. Usually then patients go to their primary care physician. They are rarely referred to hospital treatment in emergency departments. Recently, the management and detection of the causes of epistaxis have developed significantly. Therefore, effective diagnosis and treatment of epistaxis requires knowledge of the anatomy of the nose and the possible determinants of epistaxis. [15]

\section{Anatomical conditions of nose bleeding.}

The vascular anatomy of the nose is very developed and consists of numerous vascular anastomoses. The vessels are supplied from the branches of the internal and external carotid arteries. [15] The source of nosebleeds is the vast majority of the so-called Kiesselbach area, located near the anterior part of the nasal septum. It is responsible for over $90 \%$ of nosebleeds. [13] [7]. This type of bleeding is known as forward bleeding. It occurs from the richly vascularized Kissselbach plexus, which is formed by the end branches of the internal carotid artery, i.e. the anterior and posterior ethmoid arteries, and the branches of the external carotid artery, i.e. the wedge-palatal, greater palatine and upper labial arteries. [15]. In contrast, posterior epistaxis mainly originates in the branches of the wedge-palatine arteries at the back of the nasal cavity. This type of bleeding occurs at the back of the upper nasal cavity or at the back of the middle turbinate. Posterior epistaxis is dangerous because bleeding may not be visible as with frontal bleeding. Moreover, it may be asymptomatic or have less characteristic symptoms such as nausea, haemoptysis, vomiting with blood, anemia or tarry stools. [2]. 


\section{Etiology}

The causes of epistaxis can largely be determined on the basis of an appropriate history and physical examination. First of all, one should obtain information on possible causes of the onset of bleeding, previous bleeding episodes, if any, comorbidities and medications taken. It is also important to ask about environmental factors and allergies. Despite a careful history, it is often impossible to establish the causes of bleeding. [2] It has also been shown that in most cases, increased blood pressure is observed during epistaxis. However, many studies do not show a direct influence of arterial hypertension on bleeding [8]. The combination of stress and the white coat syndrome also contribute to an increase in blood pressure during bleeding. In addition, a higher percentage of nosebleeds was observed during the cold season, dry air, or as a result of significant fluctuations in atmospheric pressure and changes in ambient temperature. [9], [15], [13]. Very rarely, epistaxis may also be neoplastic, such as metastatic renal cell carcinoma. [14]. Age of patients uses drugs that affect the blood coagulation system. These preparations are also available without a prescription. Many of them, such as oral factor $\mathrm{X}$ inhibitors, warfarin, clopidogrel, non-steroidal anti-inflammatory drugs, significantly affect blood clotting and their inappropriate use leads to an increased risk of bleeding, including epistaxis. [15]. It is estimated that up to $9 \%$ of children suffer from recurrent nosebleeds. The usual source is bleeding from the front of the septum of the nose. Most of them grow out of the problem and bleeding does not occur later in life. [3.6]. For the elderly, recurrent nosebleeds that require detailed diagnosis and hospital treatment are associated with high healthcare costs. It has been found that visits to the emergency department of the elderly for nosebleeds are the most common reason why they stop taking anticoagulants (aspirin, clopidogrel). This results in a significantly increased risk of stent thrombosis, which may result in a heart attack. The elderly are also very much at risk of complications of recurrent nose bleeding, including angina, hypovolemia, aspiration or heart attack. [12].

\section{Treatment}

Correct positioning of the patient is essential to visualize the site of epistaxis. Sometimes, to obtain a good field of view, it is necessary for the patient to blow the nose and remove any residual clots. [15]. The first steps in case of epistaxis include direct pressure, application of local agents, including those that constrict blood vessels, and cauterization of the bleeding site with chemical agents or 
electrocoagulation. It is also important to fill the nose with non-absorbable materials. The greater percentage of nosebleeds is treated with nasal tampons [3]. Tranexamic acid prevents fibrinolysis by inhibiting plasminogen with fibrin. Thus, it stabilizes blood clots. Its antifibrinolytic effect is applicable in many clinical situations where it is required to reduce bleeding and prevent its recurrence. It is used in such severe cases as after cardiac surgery, where cardiopulmonary bypass has been used, or in acute bleeding from the upper gastrointestinal tract. Its properties are also used in the treatment of nosebleeds, where it is used both to stop bleeding and prevent its recurrence, and to reduce the intensity of possible re-bleeding. Tranexamic acid can reduce nosebleeds after or shortly after the first administration. This avoids the need for other interventions such as nasal pads or cauterization. Tranexamic acid can be used every few days after the first nose bleed. [11].

One of the methods is also endovascular treatment. Embolization is not typically used in anterior spontaneous epistaxis. Only in cases where symptomatic or surgical treatment has failed. Embolization, on the other hand, is indicated in posterior epistaxis, especially if the bleeding is massive, which may result in a hemorrhagic shock, or in the case of persistent bleeding despite the use of a nasal tamponade. Carotid catheterization is not necessary in the event of epistaxis. Moreover, haemorrhage from the anterior ethmoid artery is not treated endovascularly due to the risk of damage to the ophthalmic artery. In this case, surgical treatment is indicated. In the case of bilateral epistaxis, embolization of the facial and wedge-palatine arteries is allowed. [1], [10]. For a clearly visible source of frontal epistaxis, treatment with silver nitrate or electrocoagulation is effective. Before starting the procedure, it is advisable to use local anesthesia and a vasoconstrictor. According to a Swiss retrospective study, electrocoagulation was more effective than chemical coagulation in terms of therapeutic success. On the other hand, chemical cauterization is more widely available as well as easier to use and cheaper. Cauterization of the bilateral nasal septum should be avoided due to the risk of perforation. [13].

\section{Summary:}

Epistaxis is a common cause of patients visiting emergency departments. Depending on their intensity and duration, they require appropriate diagnostics aimed at identifying the source of bleeding, implementing emergency help consisting in stopping the bleeding and implementing treatment and preventing further recurrences. An important condition necessary to provide an appropriate 
solution as a consequence of the treatment selection is collecting an appropriate history, bearing in mind the multifactorial nature of this disease. Depending on the etiology and source of bleeding, a number of treatments are available. From basic ones like nasal tamponade or cauterization to endovascular procedures. The wide spectrum of treatment proves that bleeding can be dangerous to health and life, and is also a frequent reason for patients seeking medical attention.

References:

1. A.Reyre, J. Michel, L. Santini, P. Dessib, V. Vidala, J.-M.Bartolia, G. Moulina, A. Varoquauxa, Epistaxis: The role of arterial embolization Diagnostic and Interventional Imaging, Volume 96, Issues 7-8, JulyAugust 2015, Pages 757-773.

2. Corry J. Kucik, Timothy Clenney, Management of Epistaxis, Am Fam Physician. 2005 Jan 15;71(2):305-311.

3. David E Tunkel, Samantha Anne, Spencer C Payne , Stacey L Ishman, Clinical Practice Guideline: Nosebleed (Epistaxis), Otolaryngol Head Neck Surg, 2020 Jan;162(1_suppl):S1-S38.

4. Desiderio Passali, Valerio Damiani, Francesco Maria Passali, Maria Angela Tosca, Gaetano Motta, Giorgio Ciprandi, An International Survey on the pragmatic management of epistaxis, Acta Biomed, $2020 \mathrm{Feb}$ 17;91(1-S):5-10.

5. G. Meccariello, C. Georgalas, F. Montevecchi, G. Cammaroto, R. Gobbi, E. Firinu, A. De Vito, And C. Vicini, Management of idiopathic epistaxis in adults: what's new?, Acta Otorhinolaryngol Ital. 2019 Aug; 39(4): 211-219.

6. Gerald W McGarry, Recurrent epistaxis in children, BMJ Clin Evid. 2013; 0311.

7. Hasan Emre Koçak, Zeki Tolga Bilece, Mehmet Keskin, Hüseyin Avni Ulusoy, Arzu Karaman Koç, Kamil Hakan Kaya, Comparison of topical treatment methods used in recurrent anterior epistaxis: a randomized clinical trial, Brazilian Journal of Otorhinolaryngology, Volume 87, Issue 2, March-April 2021, Pages 132-136.

8. Hayoung Byun, Jae Ho Chung, Seung Hwan Lee, Jiin Ryu, Changsun Kim, Jeong-Hun Shin, Association of Hypertension With the Risk and Severity of Epistaxis, JAMA Otolaryngol Head Neck Surg, 2020 Sep 10;147(1):1-7. 
9. Jason P. Womack, Md; Jill Kropa, Md; And Marissa Jimenez Stabile, Do, Rutgers University Robert Wood Johnson Medical School, New Brunswick, New Jersey, Epistaxis: Outpatient Management, Am Fam Physician. 2018 Aug 15;98(4):240-245.

10.Joan C Wojak, Endovascular Treatment of Epistaxis, Semin Intervent Radiol, 2020 Jun;37(2):150-156.

11.Jonathan Joseph, Pablo Martinez-Devesa, Jenny Bellorini, Martin J Burton, and Cochrane ENT Group, Tranexamic acid for patients with nasal haemorrhage (epistaxis), Cochrane Database Syst Rev. 2018 Dec; 2018(12).

12. Mohamad R. Chaaban, Dong Zhang, Vicente Resto, and James S. Goodwin, Factors influencing recurrent emergency department visits for epistaxis in the elderly, Auris Nasus Larynx. 2018 Aug; 45(4): 760-764.

13.Rafael Beck, Martin Sorge, Antonius Schneider, Andreas Dietz, Current Approaches to Epistaxis Treatment in Primary and Secondary Care, Dtsch Arztebl Int, 2018 Jan 8;115(1-02):12-22.

14.Rajeev Kumar, MS, DNB, Kapil Sikka, MS, DNB, Rakesh Kumar, MS, Priti Chatterjee, MD, Nephrogenic epistaxis, Singapore Med J 2014; 55(7): e112-e113.

15.Stephanie Yau, An update on epistaxis, Volume 44, No.9, September 2015 Pages 653-656. 